\title{
DENSE KNOTS IN HIGH LATITUDE MOLECULAR
}

\section{CLOUDS}

\author{
LEO BLITZ \\ Astronomy Department, University of Maryland \\ College Park, MD, 20742 USA
}

\begin{abstract}
The high latitude molecular clouds are discussed in the context of the formation of very low mass condensations: protojupiters. The ability to identify such objects is intimately tied to the resolution of the current debate concerning the density of the condensations inferred from observations of high density tracers. At the heart of the debate is whether inclusion of electron excitation plays an important role in the determination of column densities. A brief review of molecular abundances shows that with the possible exception of $\mathrm{H}_{2} \mathrm{CO}$, the molecular abundances in the high latitude molecular clouds are not abnormal.
\end{abstract}

Key words: Interstellar:Molecules; Stars:Formation; Molecules: Abundances

\section{Introduction}

The high latitude molecular clouds (HLCs) are a diverse set of objects that encompass both dark and diffuse molecular clouds. On one end of the scale are the Barnard objects, classical dark clouds found at large angles from the Galactic midplane. Next, are the low extinction clouds found by means of their CO J = $1-0$ emission (Blitz, Magnani, and Mundy 1984). They are synonymous with the transluscent molecular clouds of van Dishoeck and Black (1989). The mean visual extinction in these clouds is $\sim 1$ magnitude, but there is a great deal of local variation. These clouds have been shown to have a mean distance from the Sun of about $100 \mathrm{pc}$, and they are, in general, not gravitationally bound. Furthermore, they are the high density cores of the infrared cirrus emission (See Blitz 1991 and references therein). A related set of objects are the "tiny little clouds," identified by Knapp and Bowers (1988) near Betelgeuse. Although they are observed closer to the plane than the HLCs identified by Magnani, Blitz and Mundy (1985), and are at the low end of the HLC size scale, their physical characteristics appear to be quite similar to the HLCs.

There are two other categories of high latitude molecular clouds identified in the literature. The first is a set of three intermediate velocity clouds (Mebold et al. 1985; Heiles, Reach and Koo 1988; Desert, Bazell and Blitz 1990) that have large negative velocities compared to the dispersion of the HLC sample. The most extensively studied of these is the Draco cloud (e.g. Mebold et al. 1985). All three may be related to material falling onto the galactic plane from the corona. The second category is a set of clouds identified from their IRAS emission to be molecular, but which contain no detectable CO J = $1-0$ emission (Blitz, Bazell, and Désert 1990). These clouds have been argued to be the classical diffuse molecular clouds seen by means 
of their dust emission. Since the diffuse molecular clouds have lower CO abundances than dark or transluscent clouds, the radio $\mathrm{CO}$ emission would be much harder to detect. At least one of them appears now to have been detected in $\mathrm{CO}$ with a long integration (Boulanger, personal communication), confirming their molecular nature.

Because even giant molecular clouds can be found far from the plane, we require clouds to satisfy two criteria in order to be classified as HLCs. First, they must be at latitudes greater than $b=25^{\circ}$, and there must be no emission contiguous with a cloud at lower latitude. In this way, one can avoid contamination of the HLCs with the high latitude extensions of large clouds located at lower latitudes.

\section{Protostars and Protojupiters}

One of the outstanding characteristics of the HLCs is their proximity. Distances have been measured to only a handful of clouds, but one of them, MBM 12, has a distance of only $65 \mathrm{pc}$, which was determined from the presence and absence of strong interstellar absorption lines in the spectra of stars of known distance along the line of sight (Hobbs, Blitz and Magnani 1986). The HLCs thus may afford us the opportunity to observe the process of star formation at the highest possible angular resolution if stars do indeed form in these clouds. In fact, the HLCs may be the best place to look for substellar masses: objects with masses less than $0.08 \mathrm{M}_{\odot}$. Sensitive searches for such "brown dwarfs" have been carried out in the infrared, but the sensitivity is considerably increased when one looks for gravitationally bound molecular condensations that have not yet collapsed to form the brown dwarfs (Pound and Blitz in prepration). With conventional single dishes, it is possible to detect molecular condensations with masses as low as the mass of Jupiter in the nearby high latitude clouds. Such "protojupiters" should be seen in abundance if the star formation efficiency in the high latitude clouds is similar to that in GMCs and if the shape of the initial mass function (IMF) is similar to a Salpeter function. Even if the the power law index of the IMF is as low as 1.0, as indicated by Kroupa, Tout and Gilmore (1991), protojupiters should still be observable in the HLCs.

The reason that the detection sensitivity of substellar masses is so high in their protojupiter phase compared to their brown dwarf phase is that in the earlier phase, the objects are much bigger and therefore are radiating through a much larger surface area. However, the search for protojupiters in the HLCs supposes that they can indeed form stars, even though the clouds as whole are not gravitationally bound. There is little reason to look for protojupiters in the HLCs unless there is already some evidence for star formation in them. The direct evidence to date is rather ambiguous. At least one of the HLCs, MBM 20, is known to have several T Tauri stars in 
its nucleus. However, this cloud is the known dark cloud L1642. Sensitive searches for young stellar objects in the HLCs that are not in the Lynds (1962) compilation (Magnani, Caillault and Armus 1990) have failed to turn up any uncatalogued stars. We must therefore ask the more fundamental question: is it possible for clouds that are not themselves gravitationally bound, to form dense cores that are bound? The answer to this question leads to a controversy which has been brewing in the study of HLCs.

To put the controversy in context, it is necssary to first describe how one would identify a protojupiter. Such objects would surely appear as dense knots in molecular maps of HLCs. To show that the mass of a candidate protojupiter is less than a stellar mass it would be necessary to get two independent measurements of the mass, both would have to agree to within the uncertainties, and both would have to show that the mass is less than $0.08 \mathrm{M}_{\odot}$. On the one hand, it would be a relatively simple task to get a virial mass estimate, from the measured linewidth and size of the condensation (assuming that it was observed in a cloud of known distance). A virial measurement is not sufficient however, because the condensation may have a much lower true mass, and the clump might be supported by pressure rather than by gravity. It is therefore necessary to obtain another measurement of the mass of the clump from measurements of several molecules and molecular transitions to establish that the clump mass is approximately equal to the virial mass. But here is where we run into trouble.

\section{Densities in the HLCs}

In a first attempt to determine molecular abundances in HLCs, Magnani, Blitz, and Wouterloot (1988) observed a number of clouds in the $6 \mathrm{~cm}$ line of $\mathrm{H}_{2} \mathrm{CO}$, and made small maps in four of the clouds. Turner, Rickard and $\mathrm{Xu}(1989)$ reobserved most of these positions in the $2 \mathrm{~cm}$ line of $\mathrm{H}_{2} \mathrm{CO}$, as well as in two lines of $\mathrm{C}_{3} \mathrm{H}_{2}$; a few positions were also observed in $\mathrm{HC}_{3} \mathrm{~N}$. These positions may be characterized as the high density cores of the HLCs. Turner et al. used the Magnani et al. results to do a multilevel analysis in both $\mathrm{H}_{2} \mathrm{CO}$ and $\mathrm{C}_{3} \mathrm{H}_{2}$ for seven of the cores and found a median value for the density in the cores of $4 \times 10^{4} \mathrm{~cm}^{-3}$. They concluded that the cores are gravitationally bound, and are very opaque with $A_{v} \sim 10 \mathrm{mag}$.

If correct, this result is very striking, because it implies that the HLCs should be active sites of star formation. Furthermore, it raises the possibility that even though both the CO and IRAS results imply that the clouds are not massive enough to be gravitationally bound, there might be enough mass in the dense cores to bind many of the unbound clouds.

Another implication is that the appearance of the clouds on the Palomar Sky Survey prints is misleading. The HLCs are very difficult to see on the Palomar prints in most cases; that is why they were, for the most part, not 
catalogued by Lynds. Star counts (Magnani and de Vries, 1986) show that the clouds have mean visual extinctions of 1 magnitude or less, yet if the Turner et al. results are correct, they should have cores, sometimes extended by as much as $10^{\prime}$, with extinctions an order of magnitude higher. There is certainly no hint from the optical appearance of the clouds that they have such dense knots, but extinction observations are on too coarse a scale to be able to make definitive judgements.

On the other hand work by van Dishoeck et al. (1991) and Gredel et al. (1991) suggests lower densities in the condensations based on multitransition studies of $\mathrm{CO}$ in a few HLC cores and observations of the important HD 210121 cloud. The latter source is particulary important because the star from which it gets is name is in the backgound and optical lines of $\mathrm{C}_{2}$ and $\mathrm{CN}$ are observed in absorption. The cloud is, however, different from most of the HLCs in that it is gravitationally bound to within the uncertainties of the measurements. In any event, van Dishoeck et al. and Gredel et al. argue that the clouds are sufficiently diffuse and transparent that electron electron excitation is important, and must be taken into account in the multitransition studies. They find that the density of the cores is likely to be significanly smaller than the values derived by Turner et al., who ignored electron excitation, by as much as a factor of about five. Unfortunately, there were no cores common to the various studies to make a direct comparison possible. The core densities derived when electron excitation is included is closer to $\sim 10^{3} \mathrm{~cm}^{-3}$; this is smaller than one would expect if the differences in the density estimates were due to electron impact alone. However, the differences between the two analyses is no longer so alarming. Furthermore, the implied extinctions are no longer at odds with the optical appearance of the clouds. Ongoing observations using different molecular tracers should resolve the issue definitively.

\section{Abundances}

It is worthwhile mentioning that at least 10 different molecules have been observed in the HLCs to date. Radio detections include $\mathrm{CO}$ (including the ${ }^{13} \mathrm{CO}$ isotope and the $\mathrm{J}=2-1$ and $3-2$ transitions), $\mathrm{OH}, \mathrm{H}_{2} \mathrm{CO}, \mathrm{CH}, \mathrm{C}_{3} \mathrm{H}_{2}$, $\mathrm{HC}_{3} \mathrm{~N}(?), \mathrm{CS}$, and $\mathrm{NH}_{3}$. Optically detected molecules include $\mathrm{C}_{2}, \mathrm{CN}$, and $\mathrm{CH}^{+}$.

The abundance of $\mathrm{CO}$ apparently varies from its value in diffuse clouds of $\sim 10^{-6}$ to the dark cloud value of $\sim 10^{-4}$ depending on the cloud density and environment (Magnani et al. 1985; Blitz et al. 1988, van Dishoeck 1991). The $\mathrm{OH}$ abundances were found by Magnani et al. (1988) to be very high $\sim 2 \times 10^{-6}$, implying a substantial enhancement, perhaps as a result of shock formation. However, Magnani and Siskind (1990) have subsequently shown that with better resolution and sensitivity, the abundance anomalies of the 
original observations disappear, and the $\mathrm{OH}$ abundances are $\sim 10^{-\tau}-10^{-8}$. Turner et al. (1989) concluded that the abundances of $\mathrm{HC}_{3} \mathrm{~N}$ and $\mathrm{C}_{3} \mathrm{H}_{2}$ were anomalously low compared to Galactic plane dark cloud values. However, if the $\mathrm{H}_{2}$ column densities they derived are too high becuase electron excitation was ignored, then the abundances of these molecules might also be normal.

There seems to be considerable disagreement on the abundance of $\mathrm{H}_{2} \mathrm{CO}$. Abundance determinations vary by four orders of magnitude ranging from $1.6 \times 10^{-6}$ (Meyerdierks et al. 1990) and $10^{-7}-10^{-7.7}$ (Grossman et al. 1990 ), to $2-3 \times 10^{-9}$ (Magnani et al. 1988 ) and $2.5 \times 10^{-9}-2.5 \times 10^{-10}$ Turner et al. (1989). The first three sets of observations are from the $6 \mathrm{~cm}$ transition alone and the last one from both the $6 \mathrm{~cm}$ and the $2 \mathrm{~cm}$ transitions taken together. Again, the Turner et al. observations are commensurate with the Magnani et al. observations if the derived $\mathrm{H}_{2}$ column densities in the former observations are too high. It is unclear why the first two sets of observations obtain such high abundances of $\mathrm{H}_{2} \mathrm{CO}$ compared to the latter two, but a consistent set of new multitransition studies sould resolve the issue.

The observational evidence therefore suggests that the chemical abundances in the HLCs are not abnormal, with the possible exception of $\mathrm{H}_{2} \mathrm{CO}$. As more molecules are observed, the physical conditions and the chemistry of these transitional objects will become more tightly constrained.

\section{References}

Blitz, L., Maganani, L, and Mundy, L. (1984), Ap. J. (Letters), 282, L9.

Blitz, L., Maganani, L, and Mundy, L. (1984), Ap. J. (Letters), 282, L9.

Blitz, L., Bazell, D., and Désert, F.X., 1988, Ap. J. (Letters), 352, L13.

Désert, F.X., Bazell, D., and Blitz, L., 1990, Ap. J. (Letters), 355, L51.

Gredel, R., van Dishoeck, E.F., de Vries, C.P., and Black, J.H., 1991, Astr. Ap, in press.

Grossman, V., Heithausen, A., Meyerdierks, H., and Mebold, U. 1990, Astr. Ap, 240, 400.

Heiles, C., Reach, W.T., and Koo, B.-C., 1988, Ap. J., 322, 313.

Hobbs, L.M., Blitz, L. and Magnani, 1986, Ap. J. (Letters), 306, L109.

Knapp, G.R., and Bowers, P.F. 1988 Ap. J., 331, 974.

Kroupa, P., Tout, C.A., and Gilmore, G. 1991, M.N.R.A.S., 251, 293.

Lynds, B.T., 1962, Ap. J. Suppl., 7, 1.

Magnani, L., Blitz, L., and Mundy, L., 1985, Ap. J., 295, 402.

Magnani, L., and de Vries, C.P., 1986, Astr. Ap, 168, 271.

Magnani, L., Blitz, L., and Wouterloot, J.G.A., 1988, Ap. J., 326, 909.

Magnani, L., Caillault, J.-P., an Armus, L. 1990 Ap. J., 357, 602.

Magnani, L., and Siskind, L. 1990, Ap. J., 359, 355.

Mebold, U., Cernicharo, J., Velden, L., Reif, K., Crezelius, C., and Georigk, W., 1985, Astr. Ap, , 151, 427.

Meyerdierks, H., Brouillet, N., and Mebold, U. 1990, Astr. Ap, 230, 172.

Turner, B.E, Rickard, L. J, and Xu., L-P., 1989, Ap. J., 344, 292.

van Dishoeck, E.F., and Black, J.H., 1989, Ap. J., 340, 273.

van Dishoeck, E.F., Black, J.H., Phillips, T.G., and Gredel, R., 1991, Ap. J., 366, 141. 
B.E.Turner: We have included electron excitation now in our models of $\mathrm{H}_{2} \mathrm{CO}$ in cirrus cloud cores, which was omitted in our earlier paper (Turner et al.1989,Ap.J.). The $\mathrm{H}_{2} \mathrm{CO}$ abundance does not change much $(\leqslant 30 \%)$ by this inclusion, but the total densities derived are reduced by a factor 3 to 5 , bringing them in good agreement with those derived from $C^{18} \mathrm{O}$ observations, and in better agreement with those derived by van Dishoeck et al.(1991), although we have studied only two clouds in common with these authors. We still find extinctions rangiong from 2 to 8 magnitudes in 17 cirrus cloud cores studied in detail, but the central densities now do not exceed $10^{4} \mathrm{~cm}^{-3}$.

L.Blitz: It remains to be seen whether the extinctions of 2-8 magnitudes are consistent, with extinction estimates from IRAS maps. I expect that 8 magnitudes in a small area will be easily visible on the palomar prints. Lowering the densities by 3-5 should now make the clumps unbound by gravity.

R.Opher: You talked about the importance of whether clouds are bound or unbound. I would like to point out that thermal instability due to bremsstrahlung radiation (e.g. Opher and Valio, 1990) would make many of the clouds, that you say are unbound, bound.

L.Blitz: I am not familiar with this work, but I doubt that a thermal instability could bind by gravity the unbound clouds. Bremstrahlung is unimportant in these objects (the ionization is low) and in any event, it's hard to see how one can cool the clouds to lower temperatures than they already are. 